\title{
Representativeness of retest participants
}

\begin{tabular}{lrrr} 
& FNR $_{\text {CMP }}$ & TPR $_{\text {PRES }}$ & TNR $_{\text {PRES }}$ \\
\hline Retest (72 examiners) & $8.8 \%$ & $31.4 \%$ & $72.9 \%$ \\
Multi42 (42 examiners) & $6.3 \%$ & $34.0 \%$ & $71.8 \%$ \\
Not retested (55 examiners) & $6.4 \%$ & $31.4 \%$ & $68.2 \%$ \\
Initial test (169 examiners) & $7.5 \%$ & $32.0 \%$ & $71.2 \%$ \\
\hline
\end{tabular}

Table S3a: Representativeness of the retest participants with respect to the 169 participants whose performance results were previously published. Three rates are shown for each group: $F N R_{C M P}$, false negative rate among all

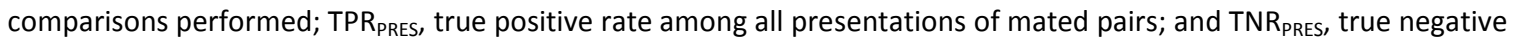
rate among all presentations of nonmated pairs. Retest participants had a notably higher false negative rate than those who were not retested. Assignment of participants to the retest or the multiple purpose test (Multi42) was based on geographic location. 